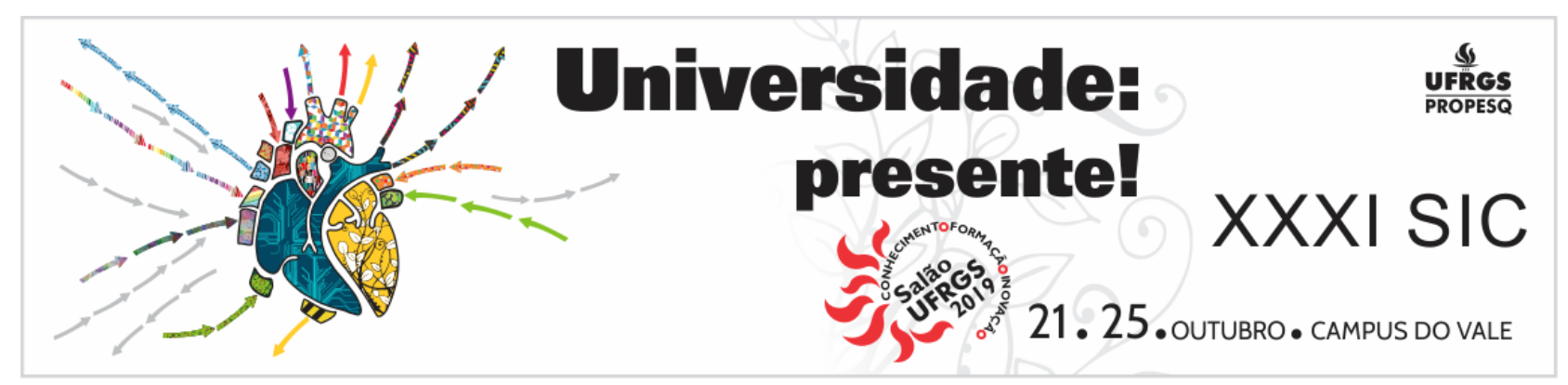

\begin{tabular}{|l|l|}
\hline Evento & $\begin{array}{l}\text { Salão UFRGS 2019: SIC - XXXI SALÃO DE INICIAÇÃO } \\
\text { CIENTÍFICA DA UFRGS }\end{array}$ \\
\hline Ano & 2019 \\
\hline Local & Campus do Vale - UFRGS \\
\hline Título & $\begin{array}{l}\text { FlowStalker: Comprehensive Traffic Flow Monitoring on the } \\
\text { Data Plane Using P4 }\end{array}$ \\
\hline Autor & LUCAS BARBOSA CASTANHEIRA \\
\hline Orientador & ALBERTO EGON SCHAEFFER FILHO \\
\hline
\end{tabular}


Universidade Federal do Rio Grande do Sul

\title{
FlowStalker: Comprehensive Traffic Flow Monitoring on the Data Plane Using P4
}

\author{
Lucas Castanheira, Alberto Schaeffer-Filho
}

\begin{abstract}
Programmability has been extensively investigated to enable a more flexible operation of computer networks. Programming a network can be achieved by either modifying its behavior in the control plane, through technologies like OpenFlow, or in the data plane, with technologies like P4. P4 is a language used to program the behavior of Software Defined Network (SDN) switches. Because it deals with software and not hardware it also drastically reduces capital expenditures and operational expenditures for the deployment of novel technologies.
\end{abstract}

With programmable data planes (PDPs) comes the possibility of revisiting many inefficient approaches to networking problems, for example how we use the data plane to create an understanding of the network state. Traditional, non-programmable switches expose only a bare minimum of what happens in their forwarding plane, forcing us to resort to inefficient methods, such as snapshotting and sampling, to acquire the state of the network. These strategies suffer from many problems, such as low accuracy of measurements and high overheads, which simply makes them unpractical for use in modern networks. We advocate that by leveraging the programmable hardware on novel programmable switches, we are able to accomplish monitoring tasks in a more efficient and comprehensive manner.

In our work, we present an efficient monitoring mechanism using programmable data planes. Our mechanism capitalizes on data plane programmability to perform tasks that are usually performed solely by the control plane (e.g. traffic monitoring and information gathering). To enable separation of concerns between the planes, we worked on two systems, firstly, a monitoring system based on a two-phase monitoring scheme that runs directly on the data plane. By running directly on switches, our monitoring system becomes much more scalable than traditional approaches. Secondly, we introduce a flexible method for network data gathering, enabling "control-plane-free" consolidation of data from switches. This is necessary because the complex computations that are done on top of monitored data should not be confined to data planes, which are too restrictive. Hence, we created an efficient system for gathering data from switches. Finally, we developed techniques for using both the monitor and the gathering system to create constant, snapshot and sampling-free analysis of network traffic. Furthermore, we evaluated our monitoring and gathering systems, showing that (1) our monitoring system is able to maintain stateful data efficiently and (2) we are able to minimize overheads of arbitrary data collection from switches when compared to other gathering techniques used on PDPs.

Our work was documented in a paper called "FlowStalker: Comprehensive Traffic Flow Monitoring on the Data Plane Using P4" which was submitted to IEEE's 53rd International Conference on Communications (ICC), a conference with the highest QUALIS ranking. The paper was accepted for publication and was presented at the conference on 22/05/2019. 